\title{
REPRESENTATION OF A CRINKLED ARC
}

\author{
RICHARD A. VITALE
}

ABSTRACT. Johnson [A crinkled arc, Proc. Amer. Math. Soc. 25 (1970), 375-376] has shown that under suitable normalizations all crinkled arcs are unitarily equivalent. Using this result, we find a general series expansion for a crinkled arc:

$$
f(t)=\sqrt{2} \sum_{n=1}^{\infty} x_{n} \frac{\sin (n-1 / 2) \pi t}{(n-1 / 2) \pi},
$$

where $\left\{x_{n}\right\}$ is an orthonormal set.

Originally introduced in problem four of Halmos [2], a crinkled arc may be defined as a continuous map $f:[0,1] \rightarrow X$, a Hilbert space, which is oneto-one and possesses the crinkly property: if $0 \leq a<b \leq c<d \leq 1$, then the chords $f(b)-f(a)$ and $f(d)-f(c)$ are orthogonal. It is convenient to consider the following normalizations:

(I) $f(0)=0$ by translation,

(II) $\|f(1)\|=1$ by a scale change,

(III) $X=\bigvee f$ where $\bigvee f$ is the smallest Hilbert space containing the values of $f$.

Under these conditions, Johnson [3] has derived a number of results including $t \rightarrow\|f(t)\|$ is a strictly monotone continuous map of $[0,1]$ onto $[0,1]$. This allows an additional normalization in the following way: if $f(t)$ is a crinkled arc with $\phi(t)=\|f(t)\|$, then $\hat{f}(t)=f\left(\phi^{-1}\left(t^{1 / 2}\right)\right)$ represents the same locus but with $\|\hat{f}(t)\|^{2}=t$. Consequently, we introduce

$$
\text { (IV) }\|f(t)\|^{2}=t
$$

and consider now only crinkled arcs satisfying (I)-(IV). In this context, Johnson's main result says that any two crinkled arcs are unitarily equivalent in the sense that if $f:[0,1] \rightarrow X, g:[0,1] \rightarrow Y$, then there is an isometry $U: X \stackrel{\text { onto }}{\rightarrow} Y$ such that $g(t)=U f(t)$. We shall use this result to prove the following representation.

Theorem. $f(t)$ is a crinkled arc iff

$$
f(t)=\sqrt{2} \sum_{n=1}^{\infty} x_{n} \frac{\sin (n-1 / 2) \pi t}{(n-1 / 2) \pi}
$$

where $\left\{x_{n}\right\} \subseteq X$ is an orthonormal set.

Presented to the Society, July 5, 1974; received by the editors June 19, 1974. $41 \mathrm{~A} 65$.

AMS (MOS) subject classifications (1970). Primary 46C05; Secondary 40J05, sion.

Key words and phrases. Crinkled arc, Brownian motion, Karhunen-Loève expan- 
The proof follows from the observation that in the theory of stochastic processes, Brownian motion $W(t)$ defined on $[0,1]$ may be regarded as a crinkled arc in a suitable Hilbert space $B$ of random variables. A direct application of the Karhunen-Loève expansion theorem provides the representation

$$
W(t)=\sqrt{2} \sum_{n=1}^{\infty} b_{n} \frac{\sin (n-1 / 2) \pi t}{(n-1 / 2) \pi}
$$

where $\left\{b_{n}\right\} \subseteq B$ is an orthonormal set (Ash [1]). If $f(t) \subseteq X$ is a crinkled arc, there is an isometry $U: B \rightarrow X$ such that

$$
f(t)=U W(t)=\sqrt{2} \sum_{n=1}^{\infty}\left(U b_{n}\right) \frac{\sin (n-1 / 2) \pi t}{(n-1 / 2) \pi} .
$$

Identifying $x_{n}=U b_{n}$, we have the desired result in one direction. Conversely, if $f(t)$ has a representation (1) then we can define an isometry $U: B \stackrel{\text { onto }}{\rightarrow} X$ coordinatewise by $U b_{n}=x_{n}$. It is immediate that all of the properties of $W(t)$ as a crinkled arc are carried into $f(t)$.

Remark 1. The series convergence in (1) is uniform in $t$ since

$$
\begin{aligned}
\left\|\sqrt{2} \sum_{n=k}^{\infty} x_{n} \frac{\sin (n-1 / 2) \pi t}{(n-1 / 2) \pi}\right\|^{2} & =2 \sum_{n=k}^{\infty}\left\|x_{n}\right\|^{2} \frac{\sin ^{2}(n-1 / 2) \pi t}{(n-1 / 2)^{2} \pi^{2}} \\
& \leq 2 \sum_{n=k}^{\infty} \frac{1}{(n-1 / 2)^{2} \pi^{2}} \rightarrow 0 \text { as } k \rightarrow \infty .
\end{aligned}
$$

Remark 2. If (IV) is dropped, then (1) holds with $t$ replaced by $\|f(t)\|^{2}$ on the right-hand side. Relaxations of (I) and (II) require the obvious modifications.

Acknowledgement. The author wishes to thank the referee for drawing attention to Johnson [4] in which an iterative construction of a crinkled arc is discussed.

\section{REFERENCES}

1. R. Ash, Information theory, Interscience Tracts in Pure and Appl. Math., no. 19, Interscience, New York, 1962. MR 37 \#5049.

2. P. Halmos, A Hilbert space problem book, Van Nostrand, Princeton, N.J., 1967. MR $34 \# 8178$.

3. G. G. Johnson, A crinkled arc, Proc. Amer. Math. Soc. 25 (1970), 375-376. MR $41 \# 4212$.

4. - Hilbert space problem four, Amer. Math. Monthly 78 (1971), 525-527. MR $44 \# 3113$.

DIVISION OF APPLIED MATHEMATICS, BROWN UNIVERSITY, PROVIDENCE, RHODE ISLAND 02912 